\title{
Oxidative stress and $\alpha$-synuclein conspire in vulnerable neurons to promote Parkinson's disease progression
}

\author{
Kelvin C. Luk

\begin{abstract}
Patients with Parkinson's disease (PD) show selective degeneration of dopaminergic neurons in the substantia nigra and cholinergic neurons in the dorsal motor nucleus (DMnX), but the drivers of this specific susceptibility are unknown. In this issue of the $J C l$, Musgrove et al. report on their use of an impressive array of in vivo and ex vivo tools for interrogating $\mathrm{DMnX}$ neurons and demonstrate that this population exhibits enhanced sensitivity to oxidative stress. Remarkably, this sensitivity was amplified by the overexpression of $\alpha$-Synuclein ( $\alpha$-Syn), a pathological protein in PD. They further show that oxidative stress augments cell-cell transfer of $\alpha$-Syn, which may be an important mechanism underlying the development and progression of PD.
\end{abstract}

Center for Neurodegenerative Disease Research, Department of Pathology and Laboratory Medicine, Perelman School of Medicine, University of Pennsylvania, Philadelphia, Pennsylvania, USA. alone in the regions examined. the striatum of wild-type mice, pointing to the selective sensitivity in the DMnX among cells expressing this neurotransmitter. However, in contrast to SNpc dopamine neurons, cholinergic neurons did not degenerate following paraquat treatment

\section{Effect of $\alpha$-Syn on oxidative stress}

Since vagal cholinergic neurons are one of the earliest populations to accumulate $\alpha$-Syn containing Lewy bodies in PD (1), the authors examined whether overexpression of $\alpha$-Syn could also modulate oxidative stress in these cells. Unilateral injection of adeno-associated virus (AAV) particles encoding human $\alpha$-Syn (h $\alpha$-Syn) into the vagus nerve increased basal ROS levels in DMnX neurons that expressed ho-Syn. While only $40 \%$ of DMnX neurons were immunoreactive for ha-Syn, oxidized DHE levels were approximately $30 \%$ higher in these cells than in neurons lacking h $\alpha$-Syn. Treatment with paraquat further increased ROS levels by 1.5 -fold and reduced the total number of surviving DMnX neurons by $25 \%$ after 7 days, suggesting a toxic synergy between oxidative stress and $\alpha$-Syn in these cells.

To test whether these combined insults in DMnX neurons also generated higher levels of modified $\alpha$-Syn typical of human synucleinopathies, Musgrove et al. probed DMnX neurons with antibodies raised against oxidized or nitrated ho-Syn. Consistent with the oxidative environment induced by paraquat and ho-Syn AAV, modified h $\alpha$-Syn could be detected in both neuronal processes and cell bodies by immunostaining and ELISA. Curiously, the accumulated $\alpha$-Syn species were also detectable with an antibody recognizing both oligomeric and fibrillar recombinant ho-Syn, but not with an $\alpha$-Syn fibrilspecific antibody (10). This differs somewhat from human PD pathology, where fibrillar $\alpha$-Syn species predominate (6), although this observation may be a func- 
tion of the short treatment durations used; whether these $\alpha$-Syn species later mature into intraneuronal pathology resembling classical Lewy bodies and neurites after prolonged exposure to oxidative conditions remains to be seen.

\section{Oxidative stress promotes $\alpha$-Syn transfer}

Although the cholinergic DMnX neurons do not project to more rostral CNS regions, they receive input from neurons in the pons and ventral midbrain (11). Previous work from the Di Monte group had shown that AAV-expressed h $\alpha$-Syn can be transferred in a retrograde fashion from the vagus to more rostral regions, such as pons and midbrain (12). In the present study, Musgrove et al. show that treatment with paraquat more than doubled the density of ha-Syn immunoreactive processes in these rostral regions. Moreover, proximity ligation assay labeling with a 3-nitrotyrosine-specific antibody confirmed that the h $\alpha$-Syn contained within these axons was nitrated, though it was unclear whether this modification occurred while in the donor (i.e., DMnX) neurons or after transfer to recipient axons. To further address this question, the authors turned to a cellbased model to measure the effect of oxidative stress on intercellular $\alpha$-Syn transfer in cocultures of two SH-SY5Y cell lines expressing complementary fragments of the Venus fluorescent protein (13). Both paraquat and hydrogen peroxide dramatically increased the proportion of cells with bimolecular fluorescence without altering total $\alpha$-Syn levels. Intriguingly, this transfer could be blocked with antibodies specifically recognizing nitrated $\alpha$-Syn. Further studies are required to resolve whether nitrated $\alpha$-Syn species are more readily released into the extracellular space or whether nitration facilitates $\alpha$-Syn uptake by neurons. Nonetheless, this observation suggests that oxidative stress enhances the cell-to-cell transmission of $\alpha$-Syn and is consistent with the in vivo finding of increased nitrated h $\alpha$-Syn in CNS regions connected to the DMnX.

What is the source of ROS in DMnX neurons following paraquat exposure?
Past studies have shown that NADPH oxidase is a potent catalyst for ROS formation from paraquat. Interestingly, mice lacking gp91phox, a subunit of the membrane-bound NADPH oxidase present in microglia, are protected against SNpc neuron loss after paraquat treatment (14). Here, Musgrove et al. demonstrated that the ROS accumulation induced by paraquat and ho-Syn overexpression was absent in the cholinergic DMnX neurons of gp91phox ${ }^{-/}$ mice, thus implicating surrounding microglia as the major source of ROS in these neurons. While this further supports the notion that the source of ROS in neurons originates from neighboring glial cells, it also raises the question of whether microglia surrounding DMnX and SNpc neurons differ functionally from other microglia or whether vulnerable neurons provide prooxidative signals to the local environment.

\section{Conclusions and future directions}

In summary, these provocative findings provide a fresh perspective on how a triad of processes frequently implicated in PD (i.e., oxidative stress, $\alpha$-Syn, and microglia) converge at a subpopulation of neurons, leading to their selective degeneration. One possible scenario is that chronic exposure to environmental toxins such as paraquat primes $\mathrm{DMnX}$ cholinergic and other vulnerable neurons, causing them to later exhibit increased $\alpha$-Syn pathology formation and dissemination. Alternatively, misfolded $\alpha$-Syn is a potent trigger for microglia, and early accumulation of Lewy pathology in DMnX neurons could trigger a neuroinflammatory cascade, leading to oxidative stress that disproportionately affects these cells (15, 16). These processes are also not mutually exclusive. Establishing the sequence in which each of these events (co-)occur will therefore be a crucial next step to translating these findings into a better understanding of human disease.

Address correspondence to: Kelvin C. Luk, 1/F Maloney Building, 3600 Spruce Street, Philadelphia, Pennsylvania 19104,
USA. Phone: 215.615.3202; Email:kelvincl@ pennmedicine.upenn.edu.

1. Braak H, Del Tredici K, Rüb U, de Vos RA, Jansen Steur EN, Braak E. Staging of brain pathology related to sporadic Parkinson's disease. Neurobiol Aging. 2003;24(2):197-211.

2. Fearnley JM, Lees AJ. Ageing and Parkinson's disease: substantia nigra regional selectivity. Brain. 1991;114 (Pt 5):2283-2301.

3. Sulzer D, Surmeier DJ. Neuronal vulnerability, pathogenesis, and Parkinson's disease. Mov Disord. 2013;28(6):715-724.

4. Dauer W, et al. Resistance of alpha-synuclein null mice to the parkinsonian neurotoxin MPTP. Proc Natl Acad Sci U S A. 2002;99(22):14524-14529.

5. Mosharov EV, et al. Interplay between cytosolic dopamine, calcium, and alpha-synuclein causes selective death of substantia nigra neurons. Neuron. 2009;62(2):218-229.

6. Spillantini MG, Crowther RA, Jakes R, Hasegawa M, Goedert M. alpha-Synuclein in filamentous inclusions of Lewy bodies from Parkinson's disease and dementia with lewy bodies. Proc Natl Acad Sci U S A. 1998;95(11):6469-6473.

7. Halliday GM, et al. Neuropathology of immunohistochemically identified brainstem neurons in Parkinson's disease. Ann Neurol. 1990;27(4):373-385.

8. Musgrove RE, et al. Oxidative stress in vagal neurons promotes parkinsonian pathology and intercellular-synuclein transfer. J Clin Invest. 2019;129(9):3738-3753.

9. McCormack AL, et al. Environmental risk factors and Parkinson's disease: selective degeneration of nigral dopaminergic neurons caused by the herbicide paraquat. Neurobiol Dis 2002;10(2):119-127.

10. Vaikath NN, et al. Generation and characterization of novel conformation-specific monoclonal antibodies for $\alpha$-synuclein pathology. Neurobiol Dis. 2015;79:81-99.

11. Kalia M, Sullivan JM. Brainstem projections of sensory and motor components of the vagus nerve in the rat. J Comp Neurol.1982;211(3):248-265.

12. Ulusoy A, et al. Caudo-rostral brain spreading of $\alpha$-synuclein through vagal connections. EMBO Mol Med. 2013;5(7):1119-1127.

13. Bae EJ, et al. Glucocerebrosidase depletion enhances cell-to-cell transmission of $\alpha$-synuclein. Nat Commun. 2014;5:4755

14. Purisai MG, McCormack AL, Cumine S, Li J, Isla MZ, Di Monte DA. Microglial activation as a priming event leading to paraquat-induced dopaminergic cell degeneration. Neurobiol Dis. 2007;25(2):392-400.

15 . Kim C, et al. Neuron-released oligomeric $\alpha$-synuclein is an endogenous agonist of TLR 2 for paracrine activation of microglia. Nat Commun. 2013;4:1562.

16. Yun SP, et al. Block of A1 astrocyte conversion by microglia is neuroprotective in models of Parkinson's disease. Nat Med. 2018;24(7):931-938. 